\title{
An evaluation of existing manual blood film schistocyte quantitation guidelines and a new proposed method
}

\author{
Tina Noutsos ${ }^{1,2,3}$, Alexandra Y. Laidman ${ }^{3}$, Lesley Survela ${ }^{4,5}$, \\ Dorra Arvanitis ${ }^{5}$, Renee Segalla ${ }^{5}$, Simon G. Brown ${ }^{6,7}$, \\ GEOFFREY K. ISBISTER ${ }^{8}$ \\ ${ }^{1}$ Menzies School of Health Research, Charles Darwin University, Darwin, NT, Australia; \\ ${ }^{2}$ College of Medicine and Public Health, Flinders University, Adelaide, SA, Australia; \\ ${ }^{3}$ Division of Medicine, Royal Darwin Hospital, Darwin, NT, Australia; ${ }^{4}$ Royal North Shore \\ Hospital, St Leonards, NSW, Australia; ${ }^{5}$ Westmead Hospital, Westmead, NSW, Australia; \\ ${ }^{6}$ Centre for Clinical Research in Emergency Medicine, University of Western Australia, Perth, \\ WA, Australia; ${ }^{7}$ Aeromedical and Medical Retrieval Division, Ambulance Tasmania, Hobart, \\ Tas, Australia; ${ }^{8}$ Clinical Toxicology Research Group, University of Newcastle, Newcastle, \\ NSW, Australia
}

\section{Summary}

Schistocytosis is the morphological hallmark of the microangiopathic haemolytic anaemia of thrombotic microangiopathy (TMA). Consensus guidelines for manual schistocyte quantitation are available, but limited research has evaluated them. The 2012 International Council for Standardization in Haematology (ICSH) recommends a schistocyte quantitation of $1 \%$ as a robust cut-off for significance, with the quantitation including helmet, crescent, triangle and keratocyte poikilocytes; and microspherocytes only in the presence of helmets, crescents/triangles, and keratocytes. We aimed to evaluate the relative contribution of these different poikilocytes to schistocyte counting; compare the ICSH method with our proposed method which counts only cells most specific for red cell fragmentation (helmet, crescent and triangular schistocytes); and evaluate inter- and intra-observer agreement. Blood films were sourced from the Australian Snakebite Project, including non-envenomed and envenomed cases, with and without TMA. In blood films across the range of schistocytosis, the predominant poikilocytes present were helmets and crescents. Triangles, keratocytes and microspherocytes were typically only present when ICSH schistocyte count was $>1 \%$. With results dichotomised as $<1.0 \%$ or $\geq 1.0 \%$, our proposed new method versus the ICSH method showed almost perfect agreement [observed agreement $95 \%$, Cohen's kappa $(\kappa)=0.84$, SE 0.04, 95\% Cl $0.76-0.92, p<0.005]$. Inter-observer strength of agreement for our method was moderate (Fleiss' $\kappa$ for comparisons between three non-unique microscopists $\kappa=0.50$, SE $0.05,95 \% \mathrm{Cl} 0.41-0.59, p<0.005)$. Intra-observer reproducibility assessed in two microscopists ranged from substantial (Cohen's $\kappa=0.71$, SE $0.08,95 \% \mathrm{Cl} 0.55-0.86$, $p<0.005$ ) to borderline almost perfect agreement (Cohen's $\kappa=0.81$, SE $0.07,95 \% \mathrm{Cl} 0.68-0.93, p<0.005)$. Schistocyte quantitation using our new method is simpler than the 2012 ICSH method and had almost perfect agreement. Our finding of moderate inter-observer agreement in quantitating helmet, triangle and crescent schistocytes is
\end{abstract}

applicable to both the ICSH and our newly proposed method. This finding underscores the importance of clinicopathological correlation and repeated examinations in the context of a clinically suspected TMA.

Key words: Schistocytes; erythrocytes; morphology; thrombotic microangiopathy.

Received 22 April, revised 31 December 2020, accepted 6 January 2021 Available online 15 April 2021

\section{INTRODUCTION}

The thrombotic microangiopathies (TMA) are a broad range of conditions with differing clinical features, aetiologies and prognoses, some of which require immediate recognition and therapy. ${ }^{1-11}$ This is best exemplified by thrombotic thrombocytopenic purpura (TTP), which is a medical emergency. TTP is caused by deficiency in a disintegrin and metalloproteinase with a thrombospondin Type 1 motif (ADAMTS13). Whilst ADAMTS13 testing is a specific confirmatory test for TTP, the turnaround time for this test is typically lengthy in routine health care settings. Current guidelines recommend commencement of immediate therapy for presumed TTP based on this presumptive diagnosis, without waiting for ADAMTS13 results.

The time critical recognition and treatment of TTP and other TMAs relies on the timely detection of red cell schistocytes on manual examination of the blood film. ${ }^{5-7,9,10,12,13}$ The unifying features of TMA are small vessel wall injury and microthrombosis, presenting with a microangiopathic haemolytic anaemia (MAHA) and thrombocytopenia., 14 Schistocytes are the morphological hallmark of the mechanical haemolysis of MAHA.,5,9,15 They appear as erythrocyte fragments and result from a mechanical haemolysis due to physical trauma or damage to erythrocytes as they transit through the microvasculature or capillary network which is obstructed by microthrombi. ${ }^{3-5,12,14} \mathrm{Al}-$ ternatives to manual blood film schistocyte quantitation by newer full blood count analysers and automated image 
analysis software have been developed. However, these methods are not recommended without manual morphological confirmation and are not in widespread routine clinical use. . $^{2,3,6,8,16}$

The finding of schistocytes on a blood film is quite specific for the diagnosis of TMA. ${ }^{2,5-7,9}$ Blood films from patients with TTP and other TMAs are variably reported to have schistocytes in excess of $0.5-1.0 \%$ of erythrocytes, ${ }^{2,3,5-9,13,17,18}$ with $1 \%$ commonly referenced as a sound meaningful threshold for clinical significance. $^{4,7,9,11,18}$ Often in TMA the degree of schistocytes is much greater than $1 \% .^{6-9,16}$ Schistocytes are not found on normal blood films, although may occur at very low frequency as an artefact due to manual fragmentation of erythrocytes during venesection or manual spreading of the blood film smear. ${ }^{2,5,9}$ Schistocytes may be seen in other conditions not related to microangiopathy, including haematological malignancy, prosthetic heart valves, megaloblastic anaemia, renal failure, iron deficiency and thalassemia..$^{5,8,10,16,18}$ In this context they are not the predominant erythrocyte abnormality, comprising only a minor component of a more global aniso-poikilocytosis. ${ }^{2,4,5,8,10,18}$ Here, the significance of schistocytes on the blood film is much reduced. ${ }^{5,8}$ When a blood film shows concurrent major morphological abnormalities consistent with these alternative diagnoses, quantitation of schistocytes is not recommended. ${ }^{4,5,18}$

The most universally accepted and specific schistocyte poikilocytes are red cells with morphological changes consistent with erythrocyte fracture lines, namely rectilinear straight edges, and sharp angles: helmet cells, crescents (including microcrescents), and triangular cells. Additional cells associated with TMA include keratocytes and microspherocytes, which are derived from schistocytes but not morphologically specific to TMA. Keratocytes are synonymous with the morphologically identical bite cells seen in oxidative haemolysis, consisting of a curved bite shaped peripheral defect in their surface. ${ }^{11}$ Microspherocytes are spherical hyperdense small poikilocytes, distinct from the rectilinear edges and sharp angles of schistocytes. They occur in autoimmune haemolysis, but may appear on blood films of patients with TMA, secondarily derived from schistocytes. $^{2-5,11,17}$

Expert working group consensus guidelines for the morphological definition of a schistocyte and methods to quantitate them are available, however there is ongoing variability in inter-laboratory practice, varied definitions of schistocyte morphology, and limited external evaluation. ${ }^{16,17,19}$ Existing scientific recommendations for the identification and standardisation of schistocyte quantitation by consensus expert groups have substantially come from French publications by the French Group of Cellular Hematology (FGCH) (Groupe Français d'Hématologie Cellulaire $)^{3,5,17}$ and more recently The Schistocyte Working Group of the International Council for Standardization in Haematology (ICSH) in 2012. ${ }^{4}$ The ICSH consensus guideline for schistocyte quantitation includes helmet cells, crescents, microcrescents, triangle cells and keratocytes. It recommends inclusion of microspherocytes in the schistocyte quantitation only in the presence of triangular/crescent, helmet schistocytes and keratocytes. Microspherocytes were not recommended for inclusion in schistocyte counting by the preceding guidelines of the FGCH. ${ }^{3,5,17}$ Few studies have externally evaluated schistocyte quantitation. ${ }^{2,6-9,16-19}$ No studies have compared quantitation methods which include or exclude the poikilocytes associated with TMA but not specific to it; keratocytes and microspherocytes; or compared ICSH and FGCH methods. Existing small studies have focused on diagnostic sensitivity and specificity, ${ }^{2,7,13}$ establishing normal reference ranges and clinical utility. ${ }^{6-9,16,18,19}$ There are few studies on reliability, inter-observer agreement and intra-observer reproducibility for differentiating schistocyte quantitation above or below the recommended $1 \%$ cut off for significance. . $^{2,6,8,18}$

Our primary aim was to evaluate the relative distribution of these different schistocyte poikilocytes in blood films from the Australian Snakebite Project for patients with and without thrombotic microangiopathy, and compare the existing schistocyte ICSH quantitation expert consensus guideline to a schistocyte quantitation method which only includes cells specific for TMA: helmet, crescent, and triangle cells. Our secondary aims were to evaluate inter-observer agreement and intra-observer reproducibility of the manual quantitation of schistocytes most specific for TMA (helmet, triangular, crescent and microcrescent cells).

\section{METHODS}

\section{Achieving standardised definitions and method of schistocyte quantitation}

An approach was developed by the authors with reference to relevant literature regarding schistocyte quantitation. French language publications were translated to English using an online translation service. Written detailed cell descriptors and blood film images with examples of representative cells were developed (see Supplementary Methods, Supplementary Figures S1-S9, Appendix A). One thousand erythrocytes were counted per examination at high power $1000 \times$ magnification under oil immersion.

Microscopists attended at least one face to face meeting and follow up virtual meetings to establish agreement and standardisation on both the morphological definition of schistocyte cell types, and training on the method of blood film cell counting. Sample blood films were reviewed for standardisation purposes at the initial face to face meeting by a multi-header microscope. Follow up virtual meetings were held for ongoing training and standardisation of schistocyte definition using blood film digital images, which included a variety of schistocytes and other poikilocytes.

\section{Source of blood films for examination}

Peripheral blood films were selected to ensure a range of degrees of schistocytosis. Slides were sourced from snakebite patients recruited to the Australian Snakebite Project. The Australian Snakebite Project is a multicentre prospective cohort study of patients with suspected snakebite with and without clinical envenoming, including cases with non-haematological envenoming syndromes, venom induced consumption coagulopathy and snakebite associated TMA. Detailed demographic and clinical information, laboratory investigations and outcomes are collected in patients from over 200 hospitals Australia wide. As part of the data collection from 2005 to 2014, serial blood films were done for patients, and then transported to the investigators. Blood films were stained by routine Romanowsky method. All blood films were deidentified to microscopists with respect to patient diagnosis; and examined blind to past or other microscopist results. All blood film examinations for both intra- and inter-observer agreement and reproducibility studies were conducted in a random order by moving the slides about in a box.

\section{Comparison of new proposed method versus ICSH consensus} guideline for schistocyte quantitation

Differential schistocyte counts were performed by the first author for helmet cells, triangular cells, crescents (including microcrescents), keratocytes and microspherocytes. The ICSH method has been previously reported, and the quantitation was derived from the combined count of helmet, crescent/ microcrescent, triangle and keratocytes; and microspherocytes included only 
if helmets, crescents/microcrescents/triangles and keratocytes were all present. Our new proposed method, which included only helmet, triangle and crescent (including microcrescent) cells, was used as a comparator.

\section{Inter-observer agreement and intra-observer reproducibility}

For inter-observer agreement studies all films were reviewed by three nonunique microscopists in total: microscopist 1 (M1), microscopist 2 (M2) or microscopist 3 (M3), and microscopist 4 (M4). Intra-observer reproducibility was studied in two microscopists (M1 and M4), who undertook a second examination of blood films.

\section{Statistical analysis}

Statistical analyses were performed using GraphPad Prism 8.2.0 for Windows (GraphPad Software, USA) except for Cohen's and Fleiss' kappa analysis which was performed in SPSS version 27.0 for Windows, (IBM SPSS Statistics, USA). Summary statistics for continuous variables were displayed as medians and interquartile ranges (IQR), and for categorical variables, as percentages.

Preliminary analyses for inter- and intra-observer studies were conducted for continuous numerical data via Bland-Altman plots. ${ }^{20}$ Based on past studies showing divergence of agreement with increasing schistocytosis, a non-linear relationship for inter-observer agreement was hypothesised. ${ }^{3,8}$ Therefore, Bland-Altman plots for both average versus difference between paired measurements, and average versus ratio of paired measurements were planned. ${ }^{20,21}$ Limits of agreement with standard error (SE) and $95 \%$ confidence intervals (CI) were determined.

Given the purpose of schistocyte quantitation to distinguish between the cut-off of $1 \%$ schistocytes, statistical analyses for comparison of the ICSH versus our new proposed method, inter-observer agreement, and intraobserver reproducibility were primarily performed as categorical data. Cohen's kappa analysis dichotomising schistocyte quantitation as $<1.0 \%$ and $\geq 1.0 \%$ was used to compare the ICSH method to our new proposed method of quantitation, paired agreement between two different microscopists, and for intra-observer studies. The Fleiss' kappa extension was used to assess inter-observer agreement between multiple three non-unique microscopists (M1, M2 or M3 and M4). The 1\% cut off was specified a priori, and prior to starting any microscopy. Cohen's and Fleiss' kappa $(\kappa)$ between $0.00-0.20$ was defined as slight agreement, $0.21-0.40$ fair agreement, $0.41-0.60$ moderate agreement, $0.61-0.80$ substantial agreement, and $0.81-1.00$ almost perfect agreement, as defined by Landis et $a .^{22}$ A $p$ value $<0.05$ was set for statistical significance.

\section{Ethics}

Human research ethics committee (HREC) approval for the Australian snakebite project was obtained from major State and Territory HRECs. Procedures followed were in accordance with the ethical standards of all relevant State and Territory HRECs and with the Helsinki Declaration of 1975, as revised in 2008. All human participants gave written informed consent.

\section{RESULTS}

\section{Comparison of ICSH method with proposed new method}

Three hundred blood films were examined with differential cell counts performed for helmet, crescent (including microcrescent), triangle, keratocyte and microspherocyte cells. Of these, six were excluded due to poor film quality, with 294 blood films included in the analysis. Differential counts showed helmet and crescent cells were the predominant poikilocytes present in all ranges of schistocytosis. Triangle, keratocyte and microspherocyte cells were proportionately less prevalent, and typically present when schistocyte count by ICSH was $>1.0 \%$ (Fig. 1). For cases with $<1.0 \%$ schistocytes by the ICSH method, median proportion of keratocytes was $0.0 \%$ (IQR $0.0-0.0 \%$ ), and median proportion of microspherocytes $0.0 \%$ (IQR $0.0-0.0 \%$ ). For cases with $\geq 1.0 \%$ schistocytes by ICSH method, median proportion of keratocytes was $0.2 \%$ (IQR $0.1-0.4 \%$ ), and microspherocytes $0.1 \%$ (IQR $0.0-0.2 \%$ ). Keratocytes were present in $15 \%$ of cases with schistocytes $<1.0 \%$ by the ICSH method, versus $94 \%$ of cases with schistocytes $\geq 1.0 \%$ by ICSH method. Microspherocytes were present in $10 \%$ of cases with schistocytes $<1.0 \%$ by ICSH method, versus $63 \%$ of cases with schistocytes $\geq 1.0 \%$

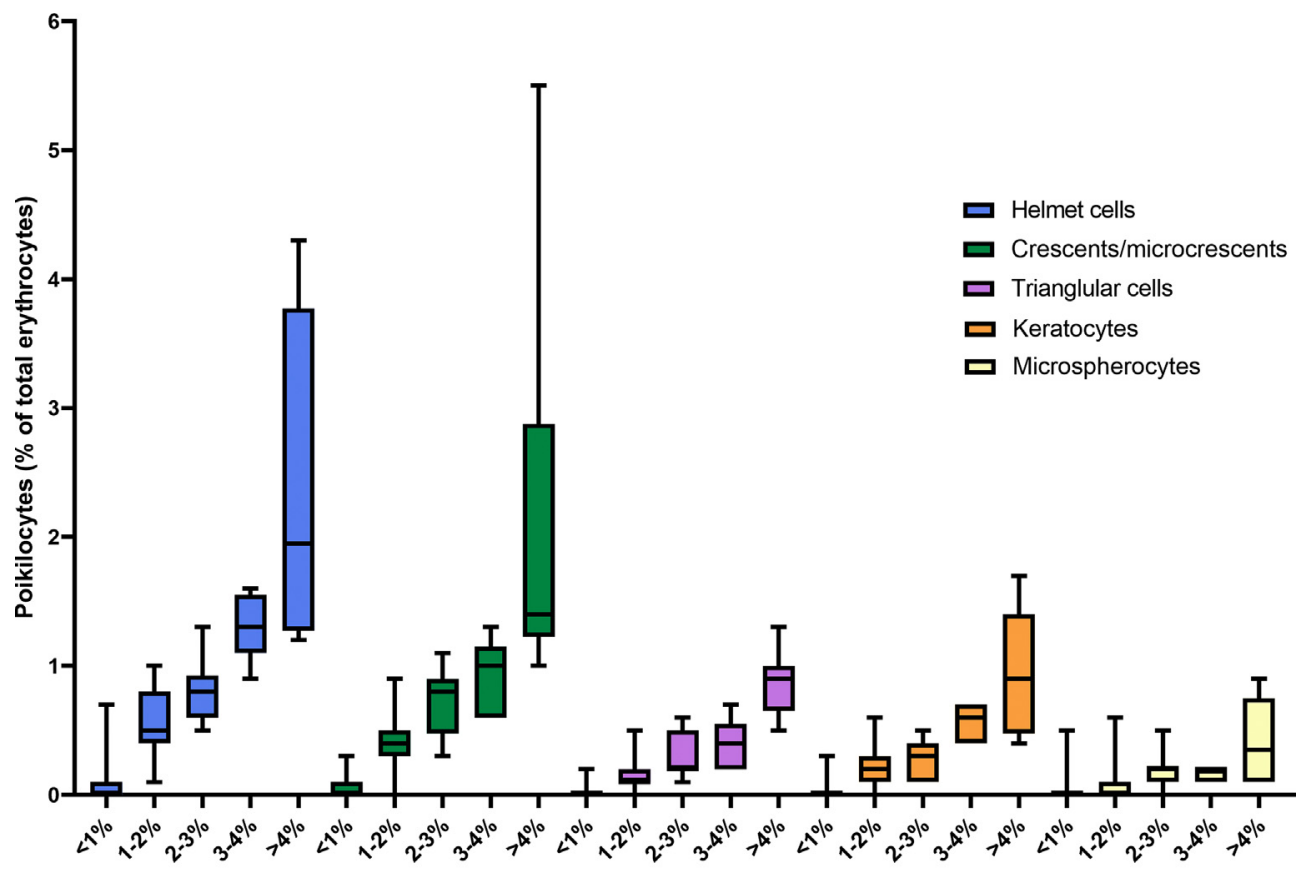

Total schistocytes by ISCH method (\%)

Fig. 1 Differential proportional counts for helmets, crescents/microcrescents, triangles, keratocytes and microspherocytes, versus International Council for Standardization in Haematology (ICSH) total schistocyte percentage. Box and whisker plot of median, IQR, minimum and maximum values. 
by ICSH method. When results were dichotomised as $<1.0 \%$ or $\geq 1.0 \%$, observed agreement between the ICSH method and our proposed new method was $95 \%$ of observations, with Cohen's kappa statistic consistent with almost perfect agreement $(\kappa=0.84$, SE $0.04,95 \%$ CI $0.76-0.92, p<0.005)$ (Table 1).

\section{Inter-observer agreement}

Two hundred blood films were examined to determine the inter-observer agreement, of which 16 films were excluded due to poor quality with significant storage artefact or crenation of cells. Of the remaining 184 films, 184 were examined by microscopist M1, 72 by M2, 112 by M3, and 156 by M4.

Paired measurements between different microscopists suggested a non-linear relationship with increasing divergence of results as the degree of schistocytosis increased (Fig. 2A-E). Bland-Altman plots for inter-observer agreement using means versus differences between paired microscopists confirmed a non-linear heteroscedastic relationship with increasing divergence in differences between schistocyte percentages as the mean percentage of schistocytes increased (Fig. $2 \mathrm{~F}-\mathrm{J}$ ). M2 versus both M1 and M4 showed a systematic bias (Fig. 2F,I), and other paired comparisons between microscopists showed a proportionate error. Inter-observer differences were much smaller for no or minimal schistocytes.

Bland-Altman plots for means of observations versus ratios of observations between M1, M3 and M4 showed a more linear relationship compared to means versus differences (Fig. 2L,M,O). Means versus ratios of observations between M1 and M2, and M2 and M4, showed wider confidence intervals and less linearity (Fig. 2K,N). For M1 and M3, bias was 1.00 (SD 0.89) and 95\% limits of agreement -0.76 to 2.75; for M1 and M4, bias 0.47 (SD 0.50) and 95\% limits of agreement -0.51 to 1.46 ; and for M3 and M4, bias 0.32 (SD 0.35) and $95 \%$ limits of agreement -0.37 to 1.01 .

Dichotomising the schistocyte count as either $<1.0 \%$ or $\geq 1.0 \%$ gave an observed agreement between paired examiners M1 versus M2, M1 versus M3, M1 versus M4, M2 versus M4 and M3 versus M4 ranging between 68\% (M1 versus M2), and 91\% (M1 versus M3). Cohen's kappa for the same paired comparisons between microscopists ranged from $0.37(0.09,95 \%$ CI $0.20-0.55)$ corresponding with a fair agreement, to 0.70 (SE $0.09,95 \%$ CI $0.52-0.87$, M1 versus M3), corresponding to substantial agreement (Table 2;

Table 1 Two by two table for International Council for Standardization in Haematology (ICSH) method versus proposed new method for schistocyte quantitation including helmet, crescent/microcrescent and triangle schistocytes only, dichotomised as $<1.0 \%$ and $>1.0 \%$

\begin{tabular}{llcc}
\hline & \multicolumn{3}{c}{ ICSH method } \\
\cline { 2 - 4 } & $<1.0 \%$ & $\geq 1.0 \%$ & Total \\
\hline New proposed method & & & \\
$<1.0 \%$ & $\mathbf{2 3 4}$ & 14 & 248 \\
$\geq 1.0 \%$ & 0 & $\mathbf{4 6}$ & 46 \\
Total & 234 & 60 & 294 \\
\hline
\end{tabular}

Bold values represent agreement between methods. Observed agreement 95\%, Cohen's $\kappa=0.84$, SE 0.04, 95\% CI 0.76-0.92, $p<0.005$.
Supplementary Tables S1-S5, Appendix A). Fleiss' kappa analysis for 159 blood films examined by three non-unique microscopists (from M1, M2 or M3, and M4) showed moderate agreement between microscopists for dichotomously categorised blood films $<1.0 \%$ or $\geq 1.0 \%, \kappa=0.50$ (SE 0.05 , $95 \%$ CI $0.41-0.59), p<0.005$.

\section{Intra-observer reproducibility}

Microscopists M1 and M4 performed blood film schistocytosis quantitations in duplicate, with their second observations blinded to their first. Paired results for each blood film together with Bland-Altman plots for average versus difference and average versus ratio of paired duplicate observations of intra-observer reproducibility for both M1 and M4 showed a more linear trend compared to inter-observer agreement (Fig. 3). For means versus ratios of observations between observations for M1, bias was 0.64 (SD 0.58) and $95 \%$ limits of agreement -0.50 to 1.79 , and for M4, bias 1.13 (SD 0.76 ) and $95 \%$ limits of agreement -0.36 to 2.61 .

Dichotomising the schistocyte count as $<1.0 \%$ or $\geq 1.0 \%$ intra-observer agreed observations were $88 \%$ for $\mathrm{M} 1$, and Cohen's kappa 0.71 (SE 0.08, 95\% CI 0.55-0.86), corresponding to substantial agreement, and for M4 agreed observations were $90 \%$, with a Cohen's kappa of 0.81 (SE 0.07, 95\% CI $0.68-0.93, p<0.005)$, corresponding to borderline substantial to near perfect agreement (Table 2; Supplementary Tables S6-S7, Appendix A).

\section{DISCUSSION}

Our study is the first to evaluate the proportionate distribution of the different types of schistocyte poikilocytes, enabling an evaluation of international expert consensus guides. The most prominent schistocytes present in quantitations were the more specific schistocytes: helmets, crescents (including microcrescents) and triangle schistocytes. The poikilocytes less specific to schistocytosis (keratocytes and microspherocytes) comprised only a minor part of the differential count, and did not significantly influence categorising schistocyte counts when dichotomised as $<1.0 \%$ or $\geq 1.0 \%$. We have proposed a new method of quantitation which includes only those schistocyte poikilocytes which are most specific to TMA. Our study findings show almost perfect agreement between the ICSH method versus our new proposed method, for determining schistocytosis at the cut-point of $1.0 \%$. This finding of near perfect agreement can also be extrapolated to the preceding French method from the FGCH group, which did not include microspherocytes as part of schistocyte quantitation.

We demonstrated overall moderate strength of interobserver agreement, and substantial to borderline near perfect strength of intra-observer agreement, for our method of schistocyte quantitation when differentiating between blood films above and below $1.0 \%$ schistocytosis. Of four unique microscopists, one showed clear evidence of systematic bias in paired comparisons with two other microscopists. The others showed a proportional error in Bland-Altman analysis, which is common in the limited other studies available on schistocyte quantitation, and in laboratory measurement studies more broadly. ${ }^{3,4,21,23}$ To our knowledge, our study is the first to evaluate inter-observer agreement together with intra-observer reproducibility. Intra-observer reproducibility is highly relevant to the study of inter-observer agreement, because the repeatability of a single microscopist's 

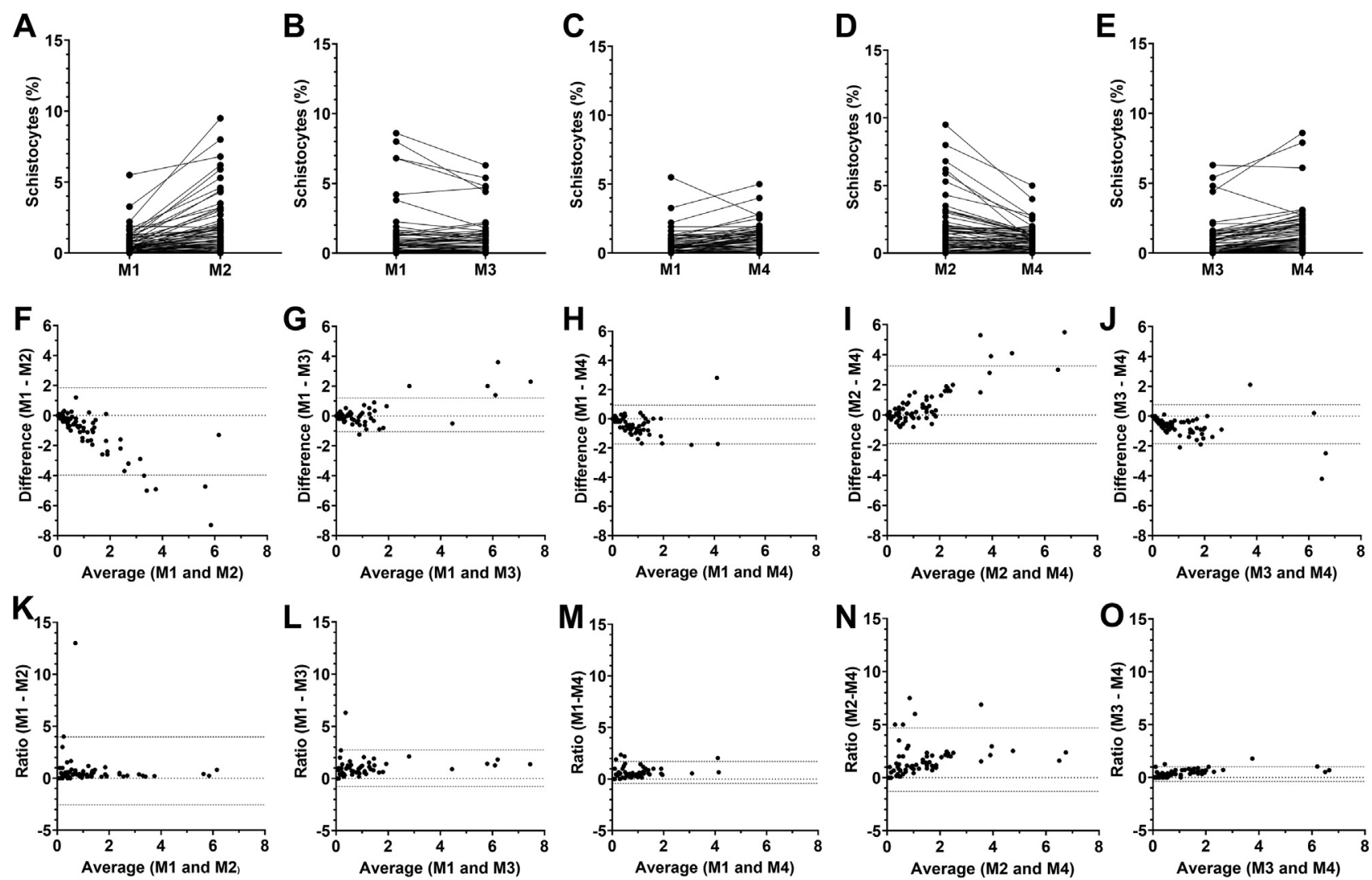

Fig. 2 Interobserver studies between microscopists for schistocyte (\%) quantitation using our proposed new method, including helmet, triangle and crescent/microscrescent schistocytes only. (A-E) Paired blood film measurements for microscopists; (F-J) Bland-Altman plots for difference versus average; (K-O) Bland-Altman plots for ratio versus average. M1, microscopist 1; M2, microscopist 2: M3, microscopist 3; M4, microscopist 4 . Dotted lines represent 0 on the y-axis and the upper and lower limits of agreement (95\% confidence interval).

measurement inherently limits the amount of possible agreement between microscopists. ${ }^{20,21,24}$ Our results support a moderate overall reliability of schistocyte quantitation. This finding is relevant to both our new proposed method, and to the existing ICSH method. Moreover, the reliance of the ICSH method on conditionally including microspherocytes in the quantitation, relies on both correctly identifying specific schistocytes, and correctly differentiating microspherocytes from spherocytes, with which there is known morphological overlap. ${ }^{4}$ This infers a potential for even greater interobserver difference, and associated measurement uncertainty.
There are multiple potential contributors to inter-observer difference in schistocyte quantitation. First and foremost is the examining microscopists' interpretation and classification of individual cell morphology that is intermediate between or does not strictly meet the criteria of schistocyte cells. ${ }^{3,8}$ Secondly, a 1000 cell count is associated with measurement error compared to higher cell counts. ${ }^{23}$ Other studies have reported more convergence in inter-observer agreement using 10,000 cell counts. ${ }^{8}$ However, higher cell counts are less practical in the diagnostic laboratory setting. The time to read a blood smear for schistocyte quantitation counting 1000

Table 2 Cohen's and Fleiss' kappa analysis for interobserver agreement for our new method of schistocyte quantitation including helmets, crescents/microcrescents and triangle schistocytes, for data dichotomised as $<1.0 \%$ or $\geq 1.0 \%$

\begin{tabular}{|c|c|c|c|c|c|c|c|}
\hline Microscopist comparisons & Films examined $(\mathrm{N})$ & Observed agreement $\%(\mathrm{n} / \mathrm{N})$ & Kappa & SE & $95 \% \mathrm{CI}$ & Kappa agreement & $p$ \\
\hline \multicolumn{8}{|c|}{ Pairwise inter-observer agreement } \\
\hline M1 versus M3 & 112 & $91 \%(102 / 112)$ & 0.70 & 0.09 & $0.52-0.87$ & Substantial & $<0.005$ \\
\hline M1 versus M4 & 156 & $72 \%(112 / 156)$ & 0.38 & 0.07 & $0.25-0.51$ & Fair & $<0.005$ \\
\hline M2 versus M4 & 68 & $84 \%(57 / 68)$ & 0.68 & 0.09 & $0.51-0.85$ & Substantial & $<0.005$ \\
\hline M3 versus M4 & 98 & $80 \%(78 / 98)$ & 0.55 & 0.08 & $0.39-0.71$ & Moderate & $<0.005$ \\
\hline \multicolumn{8}{|c|}{ Overall interobserver agreement ( 3 non-unique microscopists) } \\
\hline $\mathrm{M} 1, \mathrm{M} 2$ or M3 and M4 & 159 & - & 0.50 & 0.05 & $0.41-0.59$ & Moderate & $<0.005$ \\
\hline \multicolumn{8}{|c|}{ Pairwise intra-observer agreement } \\
\hline M1 & 96 & $88 \%(84 / 96)$ & 0.71 & 0.08 & $0.55-0.86$ & Substantial & $<0.005$ \\
\hline M4 & 82 & $90 \%(74 / 82)$ & 0.81 & 0.07 & $0.68-0.93$ & Almost perfect & $<0.005$ \\
\hline
\end{tabular}

Cohen's kappa for pairwise comparisons and Fleiss' kappa for multiple comparisons of 3 non-unique microscopists.

$\mathrm{CI}$, confidence interval; M, microscopist; SE, standard error. 
A

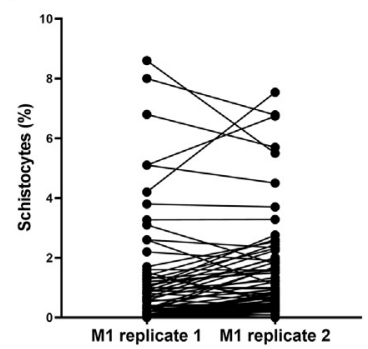

C

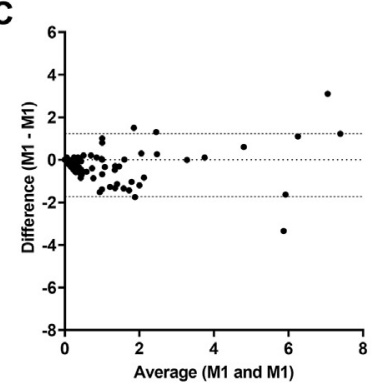

E

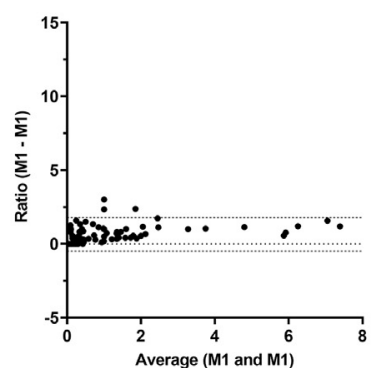

B

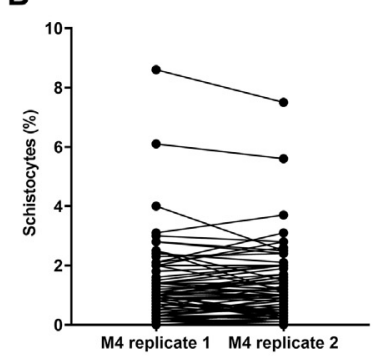

D

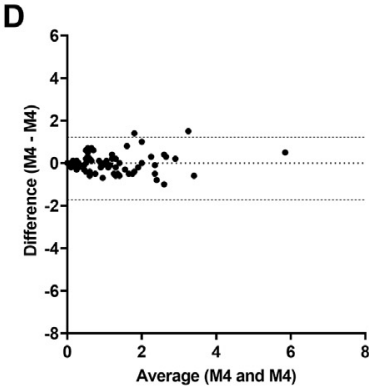

F

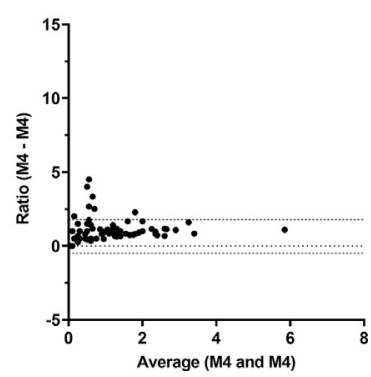

Fig. 3 Intra-observer reproducibility for replicate measurements by microscopist 1 (M1) and microscopist 4 (M4). (A,B) Paired blood film measurements for intra-observer reproducibility; (C,D) Bland-Altman plots for difference versus average and $(\mathrm{E}, \mathrm{F})$ ratio versus average. Dotted lines represent 0 on the $\mathrm{y}$-axis and the upper and lower limits of agreement (95\% confidence interval).

cells has been reported as 10.5 minutes. $^{6}$ The real world application of 10,000 cell count methods to standardise counting would be prohibitive in terms of balancing resource requirements with the potentially exhaustive resourcing required to achieve better agreement. The ICSH recommends a 1000 cell count as the best balance between the precision required versus the time and resources needed to perform a manual cell count. ${ }^{4}$

A notable strength of our study is the statistical methods used. In our scoping review of the available literature this is the first study to use Cohen's kappa statistic to evaluate inter-observer agreement categorically. Other studies have used various methods of quantitation and statistical methods for assessing agreement, predominantly Bland-Altman analysis, Pearson's R correlation coefficient and intraclass correlation coefficient (ICCC). ${ }^{2,6,8,18}$ Bland-Altman plots are fit for purpose in analysing inter- and intra-observer differences, but their meaning must be interpreted with respect to the clinical significance of the discrepancy between measurements. ${ }^{20,21,24}$ Correlation analyses also carry some limitations. Correlation examines the relationship between one variable to another, rather than also examining the differences, and is not necessarily the best approach for

studying agreement between microscopists or repeatability for a single microscopist. ${ }^{20,21,24}$ Whilst the ICCC reflects both correlation and systematic difference between measurements, it is also influenced by the range of result values. ${ }^{24}$ Arguably the clinical utility in schistocyte quantitation sits in distinguishing whether clinically significant schistocytosis is present or absent, ${ }^{5}$ and therefore categorically above or below $1 \%,{ }^{4}$ rather than the method's accuracy or reliability in distinguishing specific percentages, particularly at higher degrees of schistocytosis. Whilst any proportionate error will lead to the difference in results getting larger as the average degree of schistocytosis increases, what is clinically important is the degree of discrimination categorically at the cut-off of $1 \%$.

Our study has a few limitations. The most notable limitations include its size with respect to the number of microscopists performing the experiments, and the study population being snakebite cases only. Inter-observer studies were carried out by four microscopists, and the comparative evaluation between our proposed method and the existing ICSH by a single microscopist. Given the relative rarity of TMA, the nuanced nature of the manual quantitation of schistocytes, and recent interest in research of automated methods, it is unlikely that large and rigorously designed comparative studies or experimental studies on schistocyte quantitation methods or validation will be conducted. Our method used a 1000x magnification under oil immersion. The 2012 ICSH method recommends using medium magnifications of 400,500 or $600 \times$ magnification, using immersion oil if necessary. ${ }^{4}$ Our experience was that at both high and medium magnifications, schistocytes were easily identified and differentiated; however, cell counting was subjectively easier at higher magnifications with fewer cells per field of view, and more subtle red cell changes were better appreciated.

Our study has a number of implications for diagnostic laboratory practice. In our experience of the Australian Snakebite Project, which has recruited well over 2000 patients from more than 200 treating centres Australia wide, laboratories typically report schistocytes in a qualitative or semi-quantitative (eg.,,,,++++++++++$)$ way. We urge laboratories to start using a quantitative approach, which we endorse as within the resourcing and training capacity of routine diagnostic laboratories. Our findings of moderate inter-observer agreement underscore the importance of existing recommendations on clinicopathological correlation of results, and repeated examinations of blood films and examination via multiple microscopists where quantitation is diagnostically important, such as clinically suspected TMA with an initial negative schistocytosis blood film result. ${ }^{4,5}$ We recommend further studies in TMA of non-snakebite cases to confirm our finding of near perfect agreement between existing consensus guidelines of the ICSH and our proposed new method of including only schistocytes specific to TMA: helmet, crescent and triangle poikilocytes.

Acknowledgements: $\mathrm{TN}$ is a $\mathrm{PhD}$ candidate at the Menzies School of Health Research, Charles Darwin University, Darwin, NT, Australia. This work is submitted in partial fulfillment of the requirement for the $\mathrm{PhD}$. 
Conflicts of interest and sources of funding: The authors would like to thank the following supports for this work: TN was financially supported by a University Postgraduate Research Scholarship and Menzies School of Health Research support funds; GKI is supported by an NHMRC Senior Research Fellowship (1061041). The Australian Snakebite Project is funded by an NHMRC Clinical Centre for Research Excellence (1110343). The funders played no role in the study design, collection, analysis or interpretation of data, writing of the report, or in the decision to submit the paper for publication. The authors state that there are no conflicts of interest to disclose.

\section{APPENDIX A. SUPPLEMENTARY DATA}

Supplementary data to this article can be found online at https://doi.org/10.1016/j.pathol.2021.01.008.

Address for correspondence: A/Prof Tina Noutsos, Royal Darwin Hospital, PO Box 41326, Casuarina, NT 0810, Australia. E-mail: tina.noutsos@ flinders.edu.au

\section{References}

1. George JN, Nester CM. Syndromes of thrombotic microangiopathy. N Engl J Med 2014; 371: 654-66.

2. Lesesve JF, Salignac S, Alla F, et al. Comparative evaluation of schistocyte counting by an automated method and by microscopic determination. Am J Clin Pathol 2004; 121: 739-45.

3. Lesesve JF, Lecompte T, Alla F, et al. Reproductibility of the morphological identification of schisocytes and evaluation of non observer-dependent methods. Ann Biol Clin (Paris) 2005; 63: 279-89.

4. Zini G, D'Onofrio G, Briggs $\mathrm{C}$, et al. ICSH recommendations for identification, diagnostic value, and quantitation of schistocytes. Int $J$ Lab Hematol 2012; 34: 107-16.

5. Lesesve JF, Fenneteau O, Cynober T, et al. Role of the biologist in the study of schistocytes. Summary report and recommendations of the French Group of Cellular Haematology. Ann Biol Clin (Paris) 2003; 61: $505-12$.

6. O'Brien TE Bowman L, Hong A, Goparaju K Quantification of schistocytes from the peripheral blood smear in thrombotic thrombocytopenic purpura (TTP) compared to non-TTP thrombocytopenic hospitalised patients. Blood 2018; 132 (Suppl 1): 4983.

7. Abdelkader AM, Naguib AH, Faris MA, et al. Can schistocytes percentage in the peripheral blood smear predict cases of thrombotic thrombocytopenic purpura in women provisionally diagnosed as HELLP syndrome? Int J Reprod Med Gynecol 2018; 4: 34-40.

8. Schapkaitz E, Mezgebe MH. The clinical significance of schistocytes: a prospective evaluation of the International Council for Standardisation in Haematology schistocyte guidelines. Turk J Hematol 2017; 34: $59-63$.

9. Burns ER, Lou Y, Pathak A. Morphologic diagnosis of thrombotic thrombocytopenic purpura. Am J Hematol 2004; 75: 18-21.

10. Chabot-Richards DS, Foucar K. Does morphology matter in 2017? An approach to morphologic clues in non-neoplastic blood and bone marrow disorders. Int J Lab Hematol 2017; 39 (Suppl 1): 23-30.

11. Palmer L, Briggs $\mathrm{C}, \mathrm{McFadden} \mathrm{S}$, et al. ICSH recommendations for the standardization of nomenclature and grading of peripheral blood cell morphological features. Int J Lab Hematol 2015; 37: 287-303.

12. Sadler JE. Pathophysiology of thrombotic thrombocytopenic purpura. Blood 2017; 130: 1181-8.

13. Peedin AR, Park YA, Mazepa MA, Rollins-Raval MA, Brecher ME Raval JS. Predictive value of schistocytes in recurrence of acquired thrombotic thrombocytopenic purpura with severe ADAMTS13 deficiency at discontinuation of daily therpeutic plasma exchange. Ther Apher Dial 2018; 22: 662-5.

14. Bull BS, Kuhn IN. The production of schistocytes by fibrin strands (a scanning electron microscope study). Blood 1970; 35: 104-11.

15. Ford J. Red blood cell morphology. Int J Lab Hematol 2013; 35: 351-7.

16. Moiseev IS, Tsvetkova T, Aljurf M, et al. Clinical and morphological practices in the diagnosis of transplant-associated microangiopathy: a study on behalf of Transplant Complications Working Party of the EBMT. Bone Marrow Transplant 2019; 54: 1022-8.

17. Lesesve JF, El Adssi H, Watine J, Oosterhuis W, Regnier F. Evaluation of ICSH schistocyte measurement guidelines in France. Int $J$ Lab Hematol 2013; 35: 601-7.

18. Huh HJ, Chung JW, Chae SL. Microscopic schistocyte determination according to International Council for Standardization in Hematology recommendations in various diseases. Int J Lab Hematol 2013; 35: 542-7.

19. Ahmed M, Patel AR. Evaluation of normal reference ranges of schistocytes and burr cells in health adults. Blood 2015; 126: 4540.

20. Bland MJ, Altman DG. Statistical methods for assessing agreement between two methods of clinical measurement. Lancet 1986; 327: $307-10$.

21. Chhapola V, Kanwal SK, Brar R. Reporting standards for Bland-Altman agreement analysis in laboratory research: a cross-sectional survey of current practice. Ann Clin Biochem 2015; 52: 382-6.

22. Landis JR, Koch GG. The measurement of observer agreement for categorical data. Biometrics 1977; 33: 159-74.

23. Rumke CL. The statistically expected variability in differential leukocyte counting. In: Koepke JA, editor. Differential Leukocyte Counting. Northfield, IL: College of American Pathologists, 1979; 39-45.

24. van Stralen KJ, Dekker FW, Zoccali C, Jager KJ. Measuring agreement, more complicated than it seems. Nephron Clin Pract 2012; 120: c162-7. 